\title{
DEPLOY MECHANISM FOR VIRTUAL-MACHINE BASED VEHICULAR AD HOC NETWORK SIMULATION
}

\author{
Akihito Kohiga \\ Yoichi Shinoda \\ School of Information Science \\ Japan Advanced Institute of Science and Technology \\ Ishikawa, Japan \\ kohiga@gmail.com,shinoda@jaist.ac.jp
}

\begin{abstract}
The virtual-machine (VM) based vehicular ad hoc network (VANET) simulation executes VMs as vehicles, on which actual telecom servers and routing protocols are implemented. We would like to create massive VM based VANET simulation to verify that VANET is feasible for emergency communication facility. However, network congestion increased with the number of VMs as the ad hoc network routing protocols created multiple network sessions over a certain part of physical machines. Such network congestion limited scalability.

We propose a VM deployment mechanism to reduce network sessions over the physical machines. Our mechanism executes geographically neighboring VMs on the same physical machine. The VMs moves to another physical machine when the simulated vehicles changed positions. Our approach reduced the network sessions by approximately one-sixth. This realize the extensive VM-based VANET simulation.
\end{abstract}

Keywords: virtual machine (VM), vehicular ad hoc network (VANET) simulation, scheduling.

\section{INTRODUCTION}

Japan frequently encountered natural calamities, which disrupted the telecommunication services. For example, 1.5 million telecommunication lines were damaged during the East Japan earthquake, 2011 (NTT East 2012). Consequently, the victims suffered fatal starvation and freezing environment. Unfortunately, the "Nankai Trough earthquake" is expected soon. As the life-saving time post an earthquake is critical, working cellular network is essential for transferring information to the relevant authorities about the extent of the damage.

Vehicular ad hoc network (VANET) is apparently the most feasible alternative to damaged cellphone network. The VANET is an application of mobile ad hoc network. The telecommunication devices attached to the vehicles connect directly to the devices on other nearby vehicles. The VANET withstands natural calamities as it a) does not require stationary facilities like cellular network and b) could be powered from the vehicle power supply, which would be larger than that of a cellular phone.

Interactive simulation is effective to prove the feasibility of the VANET. Its effectiveness could be comprehended by personal hands-on experiences. Here, people experience the VANET as an alternative telecom- 
munication facility by making calls, using an actual cellphone. A basic configuration of such simulation is depicted in Figure 1. The simulation comprises of several virtual machines (VMs) as vehicles, a traffic simulator, and a WiFi emulator. The traffic simulator generates the position of each vehicle, and the WiFi emulator creates the packet drop rate, based on the vehicle position. The packet drop rate is set to the virtualnetwork card on a VM. A routing table is created by the network investigation, using optimized link state routing (Thomas Heide Clausen and Jacquet 2003) daemon, and it is the so-called ad hoc network. Here, a cellular phone user can call the other participants using cellular phone, connected to a telecommunication server on a VM (e.g., Asterisk in the figure).

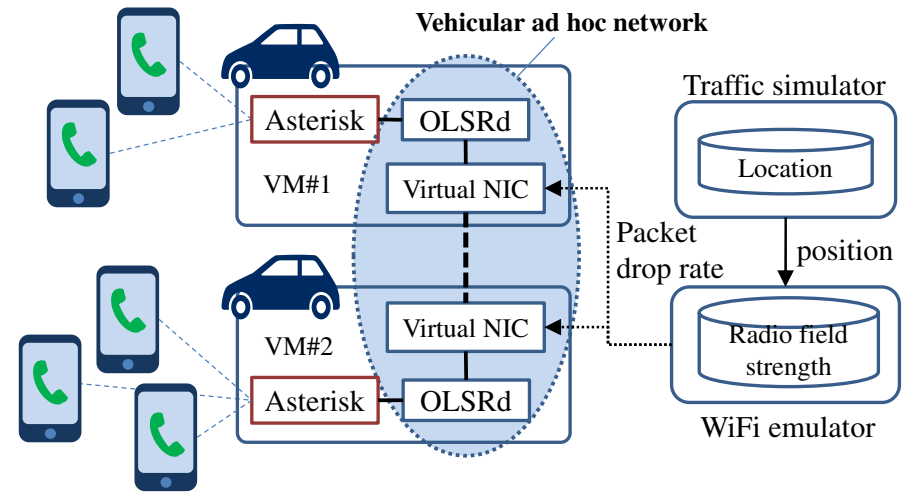

Figure 1: Vehicular ad hoc network simulation based on the VM technique.

We aim to create a scalable and interactive simulation environment to execute thousands of VMs and phone calls, using the VANET simulation. The scalable VANET simulation with actual phone-call application might give a strong impression to the authority that is concerned with disaster recovery.

\section{RELATED WORKS}

V2V, VANET Simulation: Vehicular related network simulations, which includes Vehicular to Vehicular(V2V) and VANET, verified its applicability as the smart mobility network infrastructure. VSimRTI(Schünemann 2011), Artery(Riebl, Günther, et al. 2015), iTETRIS(Rondinone, Maneros, et al. 2013) are V2V, VANET simulation frameworks. These frameworks implement APIs and the other facilities to provide interconnectivity of each component, as they assemble multiple simulation components, such as network, traffic and sensor simulator. However, framework scalability was beyond their scope. IoT simulation: Sensyml(Haris, Bisanovic, et al. 2019), iFogSim(Gupta, Vahid Dastjerdi, et al. 2017) demonstrated the IoT simulation frameworks. They created several Java-based pseudo miniature devices and analyzed the network performance. Scalability was critical for such IoT frameworks, owing to the huge numbers of IoT devices. Sensyml and iFogSim were enabled to simulate tens of thousands of clients on one PC. However, these frameworks were unable to deal with the actual IoT applications. VM-Based Network Simulation: CORE (Ahrenholz, Danilov, et al. 2008), IMUNES (Zec and Mikuc 2004), Cloonix (V. Perrier 2007) proposed the VM-based network-simulation frameworks, which comprise VMs as network nodes, virtual switches, and virtual routers in the network-topology configuration. A real network packet was transferred to a destination, through the virtual switch and router. Moreover, these frameworks could connect to a real network. However, none of these frameworks could support the ad hoc network simulation, as they aimed to make a fixed network topology and provided a test environment for network applications. Further, the scalability of these frameworks was unexplored. Traffic-Simulation Tools: SUMO (Krajzewicz, Hertkorn, et al. 2002) is one of the popular wide-area traffic simulators, which supported various traffic regulations, such as different right-of-way rules, traffic lights, different vehicle types, multi-lane streets with lane changing, and vehicle crash. We employed the SUMO simulator to generate the vehicle positions. Wireless Emulation 
Tools : ORBIT (Raychaudhuri, Ott, et al. 2005) and Qomet (Beuran, Nguyen, et al. 2007) are wireless emulation tools, which emulate the packet drop rate (or radio attenuation), following the distance between vehicles and barriers (e.g., buildings and walls). Accordingly, they calculated the packet drop rate, contained simulation maps, and simulated vehicles. The packet drop rate was set to an actual network interface device driver, on a computer.

\section{PROBLEM}

The existing VM deployment mechanism has been inappropriate for the large-scale VM-based VANET simulations. Figure 2 depicts a typical partial network congestion. Following the simulation scenario, five simulated vehicles (A-E) were located on a map. Each vehicle was connected to the other nearby vehicles, to transmit network packets. Consequently, an ad hoc network was created with its topology, which is denoted using short dash lines (Figure ??). Simultaneously, VMs (described using rectangles, A-E), which represent the simulated vehicles, were deployed using the Round Robin algorithm on PM1 to PM3.

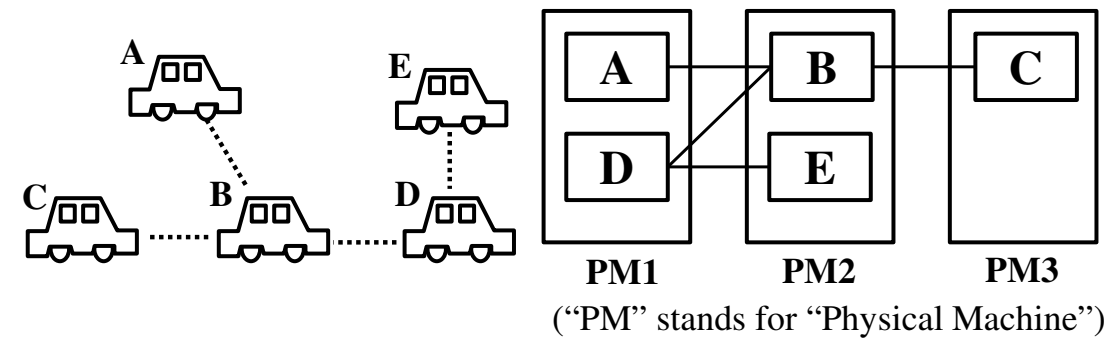

Figure 2: Local concentration of network sessions in Round Robin algorithm-based VM deployment.

Figure ?? depicts that PM1 and PM2 have considerably more network connections between them than PM2 and PM3. The existing deployment mechanism could not estimate the network topology afore. Therefore, the mechanism disregarded the ad hoc network protocol-created network topology and could not estimate the interconnectivity between the vehicles. To reduce the number of network connections over the physical machines, the VMs, B, D, and E should be executed on the same machine. However, an appropriate deployment algorithm to minimize the number of network connections between physical machines, was absent. The increase in the number of network connections between physical machines was approximately equivalent to the physical network-bandwidth saturation. The network-bandwidth saturation limited the scalability of the vehicular ad hoc network simulation. Therefore, maximal possible network connections were positioned into a physical machine.

\section{SOLUTION}

We suggest a novel VM deployment method for the VM-based VANET simulation. Our method utilizes the following characteristic of the ad hoc network: almost all the vehicles would establish a network connection with another vehicle that is running nearby to them. Therefore, we must deploy a group of VMs in one physical machine such that these VMs are "geographically" close to one another. In this section, we explain our deployment mechanism in the following four subsections.

\subsection{Geographical-Distance-Based VM Deployment}

The existing deployment approaches, such as Round Robin algorithms, result in network congestion owing to the VM deployment without network topology-related information. Conversely, none of the existing deployment mechanisms could estimate the network topology. 

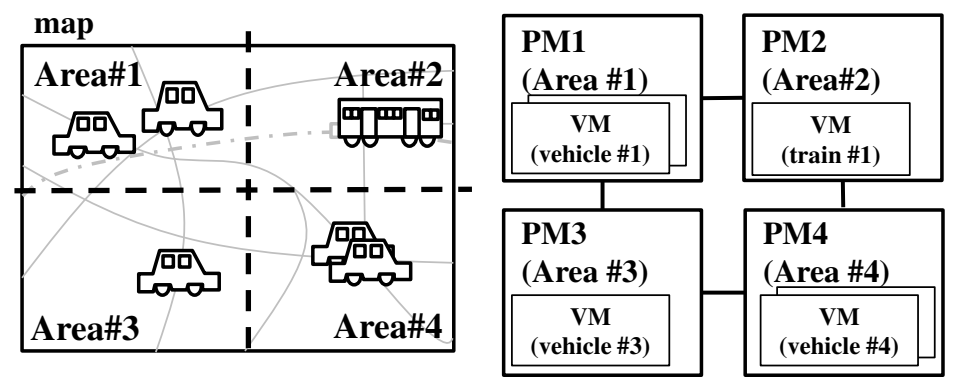

("PM" stands for "Physical Machine")

Figure 3: Dividing the simulation map into several areas and assigning them to corresponding physical machine.

The basic concept of our deployment approach is depicted in Figure 3. The left-side of the figure describes a traffic simulation and the right-side a computing cloud. In our deployment mechanism, the simulated map is divided into several parts, and one physical machine is assigned to each part. A VM, which represents a simulated vehicle, is executed on a physical machine that is assigned to the same map part to which the VM is assigned. In the figure, Area \#1 is assigned to PM1 and two VMs are executed on PM1. If a simulated vehicle crosses the boundary of the area, the VM that represents the simulated vehicle is also moved to another physical machine (typically VM live migration technique is used to move it to another physical machine).

\subsection{Voronoi Decomposition of the Simulated Map}

Separating the simulation map into subsequent areas is one of the key factors in reducing the number of network sessions between the physical machines. Vehicles stationed at a junction for longer duration than at other places. Therefore, vehicles at the junction had considerably greater number of network connections than those at other places. When the map was separated at the junction, VMs experienced greater number of connections between physical machines. This resulted in network congestion. Therefore, the junction should be kept away from the boundary of an area as far as possible.

We introduce Voronoi decomposition as a map-separation method. Voronoi decomposition is a plane partitioning method, based on the distance of each point from the boundary. In Voronoi decomposition, perpendicular bisectors is inserted between two adjacent points on a plane, which acts as the area boundaries and tessellates the plane. Moreover, all the points is equidistant from the boundary. We regard the junction on the simulation map as a Voronoi decomposition point.

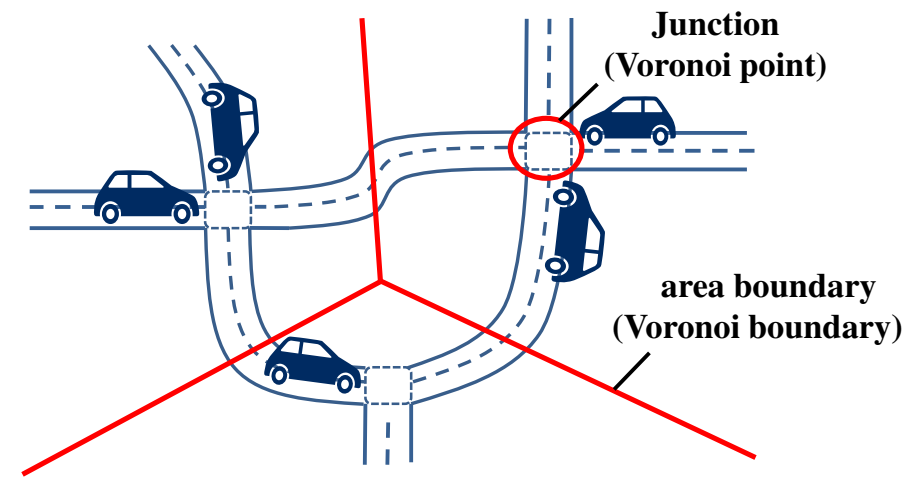

Figure 4: Voronoi decomposition based on the junction. 
Figure 4 depicts an example of a simulation map's Voronoi decomposition. We consider three junctions as Voronoi points and insert perpendicular bisectors between them. Each junction is equidistant from the area boundary. The vehicles stay at the junction for a longer duration, compared with other places, and therefore create considerably greater number of network connections around a junction. The Voronoi decomposition method can effectively put most network connections into a physical machine. Practically, we should assign a physical machine to a certain group of areas, as we cannot assign a physical machine to all junctions. We refer to a group of areas as "area cluster" in the following sections.

\subsection{Load Balancing}

A physical machine is assigned to a certain area cluster on which some VMs which represent the simulated vehicles are running. When a considerable number of simulated vehicles assemble in a certain area cluster, we must add another physical machine for load balancing. However, when all the simulated vehicles leave an area cluster, the assigned physical machine should stop working. Further, other physical machines must take over this area cluster. Our load-balancing method is presented in this subsection. We employed $10 \mathrm{VMs}$ as method implementing threshold, on a physical machine.

\subsubsection{Divide an area cluster and add a physical machine}

These following four sequences are the basic procedures to separate an area cluster and assign physical machines to the new area cluster.

i. Dividing the set of vehicles in the target area cluster into two groups using vehicle position. We utilized the $\mathrm{X}$-axis, i.e., the longitude in the simulation, for the listing, sorting, and grouping of the simulated vehicles.

ii. Dividing the area cluster into two clusters following the two vehicle groups.

iii. When two vehicles are in one area and each vehicle belongs to a different group (It is possible because two new area clusters are made on the basis of the number of simulated vehicles), This area belongs to the area cluster that have fewer simulated vehicles than those in another area cluster. However, if both the area clusters have equal number of vehicles, the area randomly belongs to any one of them.

iv. Adding and assigning a physical machine to one of the two area clusters. Only one extra physical machine must be added because one physical machine has been already presented before dividing the target area cluster.

v. Migrating the VMs to a designated physical machine. After a physical machine was added, two area clusters and two physical machines exist. However, all the VMs are executed on the previous physical machines. Therefore, half of the VMs must be moved to an appropriate physical machine following the assigned area.

\subsubsection{Breakup an area cluster and remove its physical machine}

When all the simulated vehicles left an area cluster, a physical machine that was assigned to that area cluster is not required. However, the areas in the area cluster is merged into the other area clusters, to facilitate returning vehicles.

We used a Delaunay diagram to recognize the neighboring areas, wherein each point on a plane is connected by a line to the neighboring point. The Delaunay diagram indicates the positional relationship between the 
neighboring areas in Voronoi diagram. The following sequences are implemented to split an area cluster. We referred to an area cluster that is about to be split as "AC_breakup." Moreover, the neighboring area cluster of "AC_breakup" was referred as "AC_peripheral."

i. Seeking the peripheral areas of AC_breakup, and recognizing AC_peripheral as the corresponding peripheral areas.

ii. Measuring the distances between the peripheral areas and each area in AC_breakup. Consequently, each area in AC_breakup identified its nearest peripheral area in AC_peripheral.

iii. Finally, each area is merged in AC_breakup into the AC_peripheral, the closest peripheral area.

\subsubsection{Partial exchange of area management}

Dividing an area cluster is a cumbersome task, as it involves considerable VM migration. Therefore, to reduce the complexity of the dividing task to the maximum possible extent, we introduce the partial exchange of area management. When a vehicle crosses the area cluster border, this method selectes one of the alternative plans, such as to execute VM migration or to partially exchange the areas between the area clusters. This method works as a constant load balancing method. Compared with this method, dividing an area cluster works as an urgent load balancing method.

A typical case of partial exchange is depicted in Figure 5. Vehicle\#1 is about to cross the boundary of $\mathrm{AC} 1$ and $\mathrm{AC} 2$ area clusters. Vehicle\#2 is travelling in AC2. Here, AC2 would have two vehicles if Vehicle $\# 1$ moved to AC2 and AC1 would have no vehicle. Therefore, the area should be partially exchanged in this case. The following sequences are implemented to partially exchange the areas between both the area clusters.

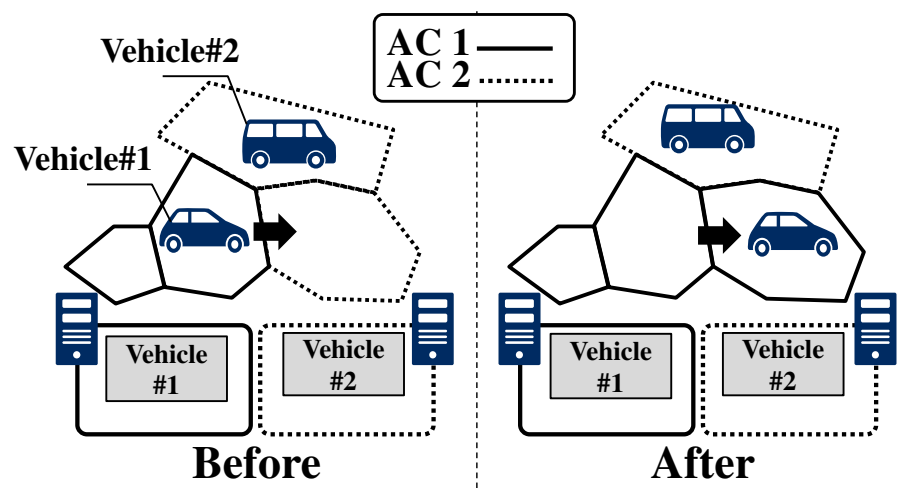

Figure 5: Partial exchange of area management.

i. When a vehicle is about to cross the boundary between two area clusters, our mechanism counts the number of vehicles in each area cluster. Our mechanism regularly monitors the vehicles' positions and watches a border crossing for making a decision whether to perform the partial exchange of areas.

ii. The subsequent two states were compared with each other. One state is obtained after the partial exchange and the other is obtained after two area clusters retained their own areas and the vehicle is migrated. Figure 5, it is evident that if the partial exchange is performed, AC1 would have one vehicle (Vehicle \#1) and AC2 would also have one vehicle (Vehicle \#2). However, if they keep their own areas, $\mathrm{AC} 2$ would have two vehicles, and $\mathrm{AC} 1$ would not have any vehicles. 
iii. One of the two states is selected. Figure 5 depicts that the target area, where Vehicle \#1 is about to enter, is partially exchanged. Consequently, the target area now belonges to AC1.

\subsubsection{Reorganize the area management}

Owing to the division, splitting an area cluster, and partial exchange of area management, our approach occasionally creates enclaves. Notably, an enclave is an area that belongs to a certain area cluster but is surrounded by areas that belong to the other area clusters. If a vehicle passed over an enclave, VM migration would be executed twice. VM migration reduces the cloud performance, therefore, enclaves must be removed as many as possible. Notably, removing enclaves results in the reduction of the number of the network connections between the physical machines and VM migrations. The reorganization of the area management must be performed as a task at constant time intervals. The following basic sequence is implemented to remove some enclaves.

i. First, the enclaves of an area cluster is recognized. If an area cluster is separated into several parts, some of them would be enclaves. The biggest area cluster is considered as the principal area. We will see some enclaves exist, when all the areas that belonged to an area cluster could not be connected in a Delaunay diagram.

ii. For each area cluster, a convex polygon of junctions is created. The polygon comprises of the outermost areas of each area cluster. This procedure is performed in the ascending order of the number of VMs.

iii. If a polygon included some areas that belong to one of the other area clusters, these areas are merged into the area cluster.

iv. The VMs in the merged areas is moved to the physical machines of the new area cluster.

v. The sequences 2 to 4 are repeated until enclave disappeared from the map.

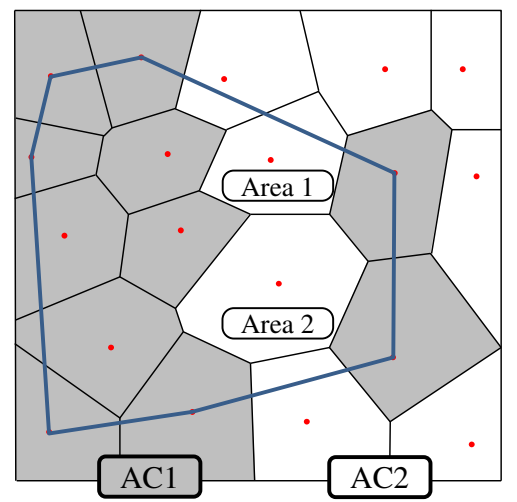

Figure 6: Enclaves and a convex polygon of junctions.

Figure 6 depicts a typical enclave removal. $\mathrm{AC} 1$ and $\mathrm{AC} 2$ are the two area clusters. The gray areas belong to $\mathrm{AC} 1$ and the white to $\mathrm{AC} 2$. Moreover, $\mathrm{AC} 1$ contains two enclaves (Area1 and Area2) while AC2 contains none. Assuming that AC1 has fewer vehicles than AC2, AC1 makes a convex polygon earlier than AC2 . Therefore, Area1 and Area2 which belong to AC2 are merged into AC1. However, when AC2 had fewer vehicles than $\mathrm{AC} 1, \mathrm{AC} 2$ made a convex polygon earlier than AC1. Accordingly, the two enclaves located at the right-side of Area1 and Area2 would be merged into AC2. 


\subsection{Simulation Architecture}

The architecture of our simulation environment comprises of a simulation manager, traffic simulator, cloud manager, WiFi emulator, and some VMs on the physical machines (PM\#1-N in the figure 7). The traffic simulator is executed, which produced the positions of each vehicle at every time point Subsequently, the map and the position of each vehicle is input to the simulation manager and WiFi emulator. The WiFi emulator calculates the packet drop rate of each vehicle at every time point and sets on the network interface card for each VMs. Simultaneously, the simulation manager constructs a Voronoi diagram, using the map and configures several area clusters. Notably, the number of area clusters depends on the number of physical machines to be used during initialization. Subsequently, these physical machines are assigned to each area cluster. Furthermore, the simulation manager requests a cloud manager, for VM deployment/migration, in accordance with the position of each vehicle at every time point. After the cloud manager deployed a VM, the IP address of each VM is informed to the WiFi emulator, to identify the vehicles with their VMs in the traffic simulation.

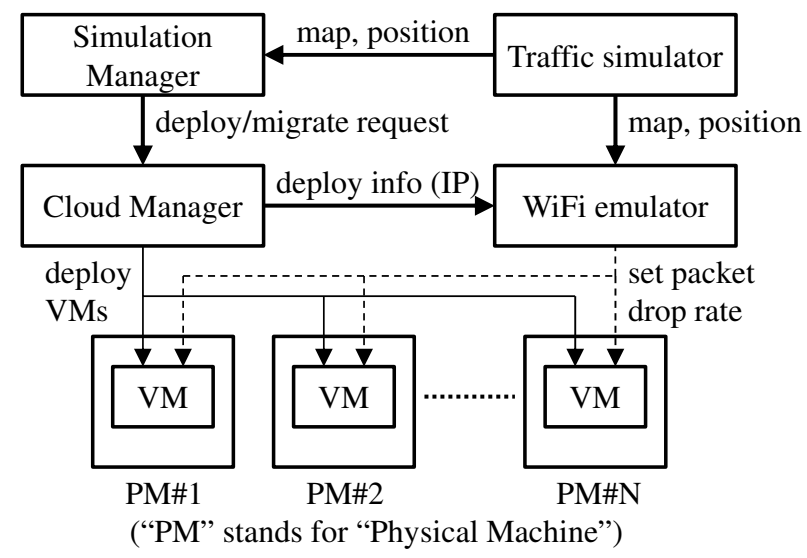

Figure 7: Simulation architecture.

\section{EVALUATION}

We suggested a VM-deployment mechanism that reduced the number of network connections between physical machines. Compared with the existing deployment mechanisms, our approach uses physical machines to a higher extent as our approach do not always pack VMs maximally into physical machines. Therefore, our approach should be evaluated based on the basis of the following two points. Compared with the existing deploy mechanisms, our approach exhibits 1) reduction in the network connections between physical machines and 2) number of physical machines required upon using our approach. The evaluation environment was presented followed by the outcome of the above-mentioned two evaluation points here.

\subsection{Evaluation Circumstances}

Table 1 lists the programs used in the evaluation. A simulation manager and mock-up modules that represented a WiFi emulator, Cloud manager, VM, and physical machines were created. Moreover, the SUMO traffic simulator was employed to create the input data (a map and the position of each vehicle).

We must reveal the ideal number of network connections, between physical machines, that can be reduced using our approach. Therefore, the evaluation of our approach was isolated from the actual cloudmanagement operation. To evaluate our approach, without depending on a specific cloud management, we 
Table 1: Programs to be used for evaluation.

\begin{tabular}{|l|l|}
\hline Simulation Manager & Our program \\
\hline Traffic Simulator & SUMO traffic simulator \\
\hline WiFi Emulator, Cloud Manager, VM and physical machine & Mock-up module on our program \\
\hline
\end{tabular}

omitted actual cloud environments, such as OpenStack and Kubernetes. However, our mock-up module for VM and physical machines could be easily replaced by an actual module.

The parameters used by the SUMO traffic simulator are listed in Table 2. The SUMO traffic simulator required a map and vehicle information (appealing place and route) as the input. A grid-shaped loadable map and vehicle information was generated using the SUMO simulator. A maximum of 1000 vehicles were generated at the beginning of the simulation, and they were mobilized randomly until the end of the simulation.

Table 2: Traffic-simulation parameters.

\begin{tabular}{|l|l|}
\hline map & $2 \mathrm{~km} \times 2 \mathrm{~km}$ square, 400 junctions, two-way loads \\
\hline number of vehicles & 100 to 1000 vehicles \\
\hline simulation time & $200 \mathrm{~s}$ \\
\hline each vehicles emersion & beginning of the simulation \\
\hline route of each vehicle & Random \\
\hline
\end{tabular}

\subsection{Result(Physical-machine utilization)}

The evaluation of the physical-machine utilization is depicted in Figure 8. Notably, the x-axis represents the elapse of the simulation time and y-axis, the number of physical machines used in the simulation. This evaluation compared ours and the complete-packing approaches. Notably, complete packing approach is a deployment approach that packs VMs into each physical machine, till the upper limit of the VM number, on one physical machine. Therefore, an ideal situation for CPU-resource utilization was depicted, The upper limit was at $10 \mathrm{VMs}$ in our approach. Therefore, if $10 \mathrm{VMs}$ are executed on one physical machine, another physical machine will be used for the next VM. This simulation indicated that our approach employed considerably more physical machines than the complete-packing approach. Especially, our approach used physical machines about 2.5 times higher than that used by the complete-packing approach.

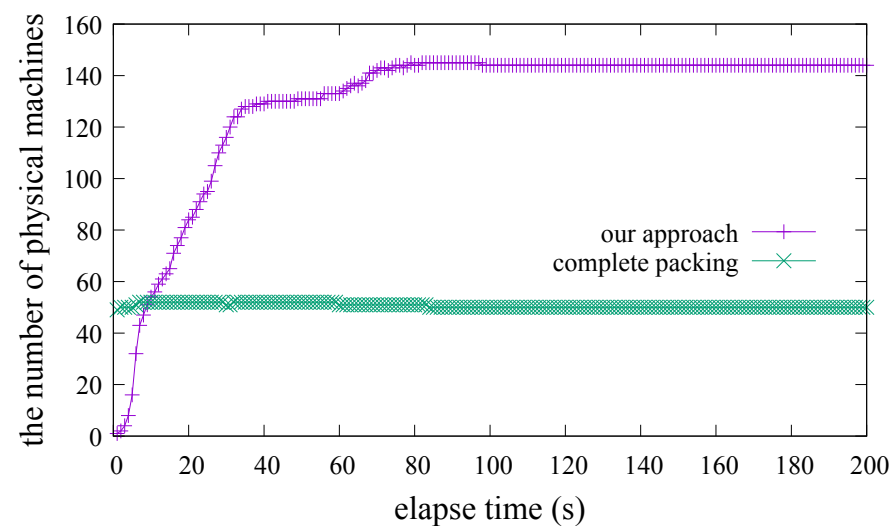

Figure 8: Number of physical machines used to execute 500 VMs. 
This gap between ours and the complete-packing approach could be rationalized as our approach a) neither always utilized the physical machine to the maximum extent and b) nor split an area cluster and removed the physical machine until there were no vehicles in that area cluster. Delaying until the number of moving vehicles in an area cluster become zero was simple. Moreover, if our approach included the VM number as a lower threshold, to split the area cluster. A certain number of VM migrations would be executed, thereby, consuming additional CPU time. A trade-off existed between the number of VM migrations and utilization of the physical machine.

\subsection{Result(Number of sessions between physical machines)}

Figure 9 depicts the relationship between the simulation elapsing time and the number of network sessions between the physical machines. This compared between Round Robin, complete packing, and our approach, for $500 \mathrm{VMs}$. Evidently, the characteristics of Round Robin and complete packing were similar during the simulation, with approximately 300 constant network sessions during the simulation. However, in our approach, the number of network sessions reached approximately 50 during the simulation. Therefore, our approach reduced the number of network sessions over the physical machine, by approximately one-sixth.

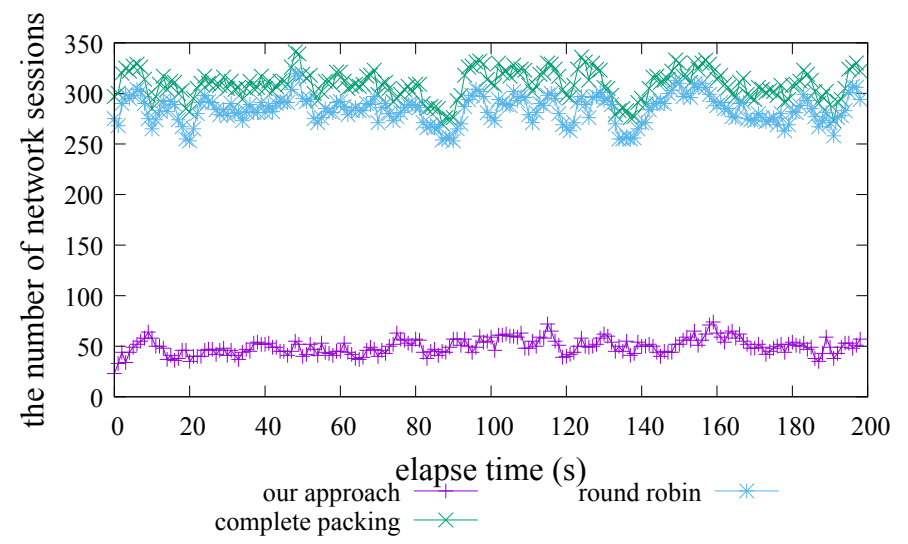

Figure 9: Elapse of the number of network sessions per second (the number of executed VMs is 500).

The relationship between the number of VMs and the number of network sessions is depicted in Figure 10. Notably, the $\mathrm{x}$-axis represents the number of VMs and y-axis, the total number of network sessions over the physical machines. For approximately 500 VMs in the figure, the existing approaches, Round Robin, and complete packing approaches generated the network session six times more than our approach. The above-mentioned approaches generated network sessions exponentially. However, our approach controlled the increase in network sessions in almost a linear manner. Therefore, compared with the other approaches, our approach reduced the number of network sessions.

\section{DISCUSSION}

\subsection{Parameter Optimization}

Two optimizations are needed for our approach. A) to select a lower threshold for splitting an area cluster. Our approach delayed the split of an area cluster until there is no vehicle in the area cluster (see Section 4.3.2), and consequently resulted in lower CPU utilization. However, with a certain number of VMs as a lower threshold, our approach could execute the same number of VM migrations that would lower the cloud's performance. We need to identify an optimal threshold to breakup an area cluster. B) to divide one 


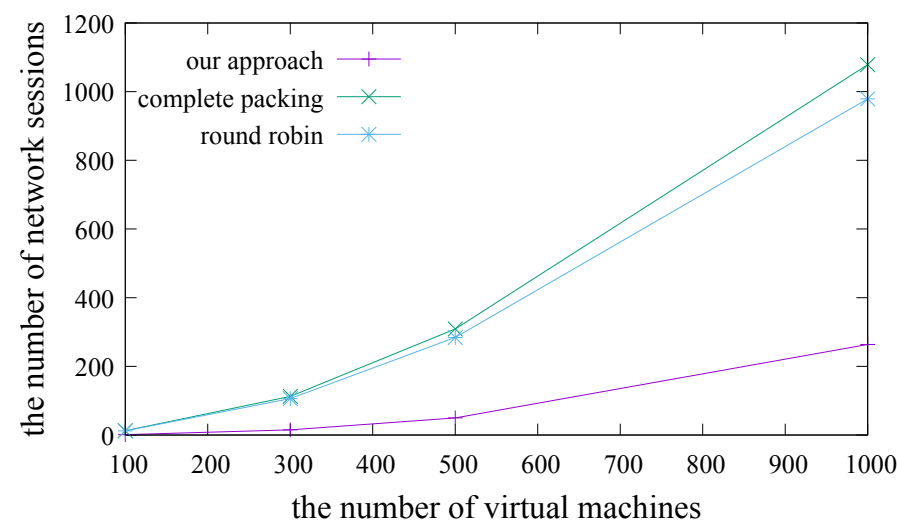

Figure 10: Total number of network sessions.

area cluster into two. Dividing an area cluster and adding a physical machine (see Section 4.3.1) resulted in a certain number of VM migrations. If two VMs, one of which is about to migrate to a new physical machine, and the other remains at the current physical machine, have a network connection, such division would result in more network sessions over the physical machines. We used x-axis to divide a group of VMs into two groups. A method to minimize the network connections over the physical machines is required.

\subsection{Another Application}

We implemented VM technology-based scalable VANET simulation as a target application, to demonstrate the feasibility of VANET as an alternative method for damaged telecommunication facilities during the initial lifesaving hours after a natural calamity. Meanwhile, Vehicle to Vehicle (V2V) or Vehicle to Infrastructure (V2I) connections would be another application of our simulated environment because the locality of the network connection exist and none of the existing deployment approaches can estimate the network connections between the vehicles. Our mechanism could be applied to V2V and V2I simulation, in the future.

\section{CONCLUSION}

We proposed a VM deployment mechanism based on the Voronoi decomposition of a simulated map. Our mechanism executed several geographically closer VMs on the same physical machine. compared with the complete-packing and Round Robin deployment approach, our approach reduced the number of network sessions by one-sixth for $500 \mathrm{VMs}$. We demonstrated that our mechanism contributes to realize a scalable VANET simulation and will be useful for the authority that concern disaster recovery.

\section{REFERENCES}

Ahrenholz, J., C. Danilov et al. 2008, Nov. "CORE: A real-time network emulator". In MILCOM 2008 2008 IEEE Military Communications Conference, pp. 1-7.

Beuran, R., L. T. Nguyen et al. 2007, May. "QOMET: A Versatile WLAN Emulator”. In 21st International Conference on Advanced Information Networking and Applications (AINA '07), pp. 348-353.

Gupta, H., A. Vahid Dastjerdi et al. 2017. "iFogSim: A toolkit for modeling and simulation of resource management techniques in the Internet of Things, Edge and Fog computing environments". Software Practice and Experience vol. 47 (9), pp. 1275-1296. 
Haris, I., V. Bisanovic et al. 2019. "Sensyml: Simulation Environment for large-scale IoT Applications". IECON 2019 - 45th Annual Conference of the IEEE Industrial Electronics Society vol. 1, pp. 30243030.

Krajzewicz, D., G. Hertkorn et al. 2002. "SUMO (Simulation of Urban MObility) - an open-source traffic simulation”. In 4th Middle East Symposium on Simulation and Modelling, pp. 183-187.

NTT East 2012. "Recovering from the Great East Japan Earthquake: NTT East's Endeavors". Technical report.

Raychaudhuri, D., M. Ott et al. 2005, Feb. "ORBIT radio grid testbed for evaluation of next-generation wireless network protocols". In First International Conference on Testbeds and Research Infrastructures for the DEvelopment of NeTworks and COMmunities, pp. 308-309.

Riebl, R., H. Günther et al. 2015, June. "Artery: Extending Veins for VANET applications”. In 2015 International Conference on Models and Technologies for Intelligent Transportation Systems (MT-ITS), pp. 450-456.

Rondinone, M., J. Maneros et al. 2013. "iTETRIS: A modular simulation platform for the large scale evaluation of cooperative ITS applications". Simulation Modelling Practice and Theory vol. 34, pp. 99 125.

Schünemann, B. 2011. "V2X simulation runtime infrastructure VSimRTI: An assessment tool to design smart traffic management systems”. Computer Networks vol. 55 (14), pp. 3189 - 3198. Deploying vehicle-2-x communication.

Thomas Heide Clausen and Jacquet, Philippe 2003. "Optimized Link State Routing Protocol (OLSR) RFC3626". https://tools.ietf.org/html/rfc3626.

V. Perrier 2007. "Cloonix”. http://clownix.net/.

Zec, M., and M. Mikuc. 2004, 01. "Operating system support for integrated network emulation in imunes". 1st Workshop on Operating System and Architectural Support for the on Demand IT InfraStructure (OASIS), pp. 3-12.

\section{AUTHOR BIOGRAPHIES}

AKIHITO KOHIGA is a Project Researcher of Information Sciences at Japan Advanced Institute of Science and Technology. His research interests lie in cloud computing and massive distibuted simulation modeling and architecture, especially in mobility and self driving. His email address is kohiga@gmail.com.

YOICHI SHINODA is a Distinguished Professor of Information Sciences at Japan Advanced Institute of Science and Technology. He holds a Ph.D. in Engineering from Tokyo Institute of Technology. His research interests include the impact of digital technologies on human activities, parallel and distributed systems, and the networking protocols and systems. His email address is shinoda@jaist.ac.jp. 